\title{
Self-contained Antenna Crosstalk and Phase Offset Calibration by Jointly Solving the Attitude Estimation and Calibration Problem
}

\author{
S. Zorn ${ }^{1}$, M. Niestroj ${ }^{1}$, S. Caizzone ${ }^{2}$, M. Brachvogel ${ }^{1}$, M. Meurer ${ }^{1,2}$ \\ ${ }^{1}$ Chair of Navigation, RWTH Aachen University \\ ${ }^{2}$ Institute of Communications and Navigation, German Aerospace Center (DLR), \\ Oberpfaffenhofen, Germany \\ Email: soeren.zorn@nav.rwth-aachen.de
}

\section{BIOGRAPHIES}

Soeren Zorn received his M.Sc. in electrical engineering from RWTH Aachen University in 2015. His bachelor and master thesis covered spoofing detection and mitigation. He is currently pursuing his Ph.D. at RWTH as a member of the Chair of Navigation. His research interests include multi antenna GNSS receivers, attitude and calibration estimation, robust interference and spoofing detection and mitigation.

Michael Niestroj received his M.Sc. in electrical engineering from RWTH Aachen Universtiy in 2016. His master thesis covered attitude and calibration estimation for multi antenna GNSS receivers. He is currently prusuing his Ph.D. at RWTH Aachen University as a member of the chair of Navigation. His research is focused on calibration methods for GNSS multi antenna systems.

Marius Brachvogel received his M.Sc. degree in electrical engineering from the RWTH Aachen University in 2016. Since then, he works as a research assistant for the Chair of Navigation. His current research includes GNSS interference and spoofing mitigation on distributed antenna systems.

Stefano Caizzone rceived the M.Sc. in Telecommunications Engineering and the Ph.D. degree in Geoinformation from the University of Rome Toer Vergata, Italy, in 2009 and 2015, respectively. Since 2010, he is with the Antenna group of the Institute of Communications and Navigation of the German Aerospace Center (DLR), Wessling, Germany, where he is responsible for the development of innovative miniaturized antennas. His main research interests concern small antennas for RFIDs and satellite navigation, antenna arrays and grids with enhanced sensing capabilities, controlled radiation pattern antennas for robust satellite navigation and high-performance antenna design for precise satellite navigation. He is a participant of RTCA SC-159, active in the definition of minimum operational performance specifications for avionic GNSS equipment.

Michael Meurer received the diploma in electrical engineering and the Ph.D. degree from the University of Kaiserslautern, Germany. After graduation, he joined the Research Group for Radio Communications at the Technical University of Kaiserslautern, Germany, as a senior key researcher, where he was involved in various international and national projects in the field of communications and navigation both as project coordinator and as technical contributor. From 2003 till 2013, Dr. Meurer was active as a senior lecturer and Associate Professor (PD) at the same university. Since 2006 Dr. Meurer is with the German Aerospace Centre (DLR), Institute of Communications and Navigation, where he is the director of the Department of Navigation and of the center of excellence for satellite navigation. In addition, since 2013 he is a professor of electrical engineering and director of the Chair of Navigation at the RWTH Aachen University. His current research interests include GNSS signals, GNSS receivers, interference and spoofing mitigation and navigation for safety-critical applications. 


\begin{abstract}
Array processing algorithms such as direction of arrival (DOA) estimation, deterministic beamforming and null-steering as well as spoofer detection and mitigation are known to be sensitive to errors in the model for the antenna array spatial response. In particular, unknown gain and phase mismatches between antenna channels, mutual coupling between antenna elements, crosstalk-effects in the RF-Frontend and in the case of using ephemeris data as auxiliary spatial information unknown antenna array attitude can seriously degrade the performances of array-processing algorithms. This paper proposes a novel algorithm to estimate gain, phase and mutual coupling / crosstalk coefficients as well as the attitude of the antenna array by exploiting the ephemeris from each GNSS satellite that is sent along with the navigation data. The estimation of the various parameters is based on a maximum likelihood approach. Numerical results are shown to illustrate the potential usefulness of the proposed calibration algorithm toward better accuracy and resolution in deterministic array-processing algorithms.
\end{abstract}

\title{
INTRODUCTION
}

Regarding positioning, navigation and timing in safety critical applications, GNSS array antenna systems currently provide the most effective way to suppress Radio Frequency Interferences (RFIs), spoofers and multipath signals [1]. Moreover, attitude determination using array antenna systems is attracting significant attention. However, for the underlying algorithms to operate properly precise antenna calibration is often a mandatory requirement. It turns out that antenna calibration is still a major challenge. Therefore many research studies came up with so-called blind interference and / or spoofing countermeasures, that do not rely on calibration [1-3]. Although they are not complicated in terms of implementation and complexity and provide good results in most scenarios, they may degrade receiver performance by accidentally nullifying or attenuating authentic signals. Beside that, these methods can severely be improved by calibration using deterministic beamforming and / or utilizing the exact steering vector, which is known through rotating the DOAs obtained from the ephemeris for each satellite by the antenna attitude. Other methods heavily depend on calibrated antenna arrays as for example the spoofing detection algorithm presented in [4], that will exclude PRN-Codes from Position-Velocity-Time (PVT) calculation, if the difference between the estimated and the expected DOA is too large.

Most of the deterministic algorithms or the DOA estimator assume a perfectly known antenna array manifold. However, in practice there are several imperfections, that have to be considered. At the level of the antenna array, mutual coupling between the antenna elements occurs. Mutual coupling is proportional to the inverse of the distance between the antenna elements and is therefore often a problem for miniaturized antenna arrays. It can be reduced by means of adding a decoupling and matching network [5], by using a split ring resonator (SRR) [6], or by using metallic fences between the antenna elements [7,8]. At the same level, there are also gain and phase mismatches introduced by the individual direction dependent radiation characteristics of each antenna element. Both of the former imperfections are known to be stable over time and can therefore be measured in an anechoic chamber during the manufacturing process. On the other hand, active elements like amplifiers, downconverters as well as various filters and unequal cable lengths also introduce gain and phase mismatches among the antenna channels. Moreover, there are crosstalk-effects present due to substrate coupling, magnetic mutual coupling between inductors, magnetic and capacitive coupling between signal lines, imperfect ground and DC lines [9], coupling due to reflection as well as various spatial filters. Those imperfections were shown to vary over time due to mechanical and thermal effects and have to be compensated during runtime [10]. Therefore, those are termed time-varying contributions in the following.

Due to the reasons above, self-calibration or online calibration techniques have aroused much interest in the past few decades [10-20]. In [10] Konovaltsev et al. suggest to generate a GNSS like calibration signal, which is injected into the antenna elements. After correlating with the corresponding signal copy the received signal vector represents the gain and phase mismatches between the antenna channels. Alternatively, one can make use of the incoming signals to calibrate the antenna array. Nearly three decades ago Friedlander at al. already discuss a technique based on the eigendecomposition of the sample covariance matrix to estimate DOAs for the incoming signals, the gain and phases of each sensor and the mutual coupling coefficients [11]. In [12] the various parameters are instead estimated based on a maximum likelihood approach using sources in known locations. In [13] Sellone et al. extend the maximum likelihood approach without the need for sources in known locations. In $[14,15]$ the unknown coefficients are estimated based on the fact that signal and the noise subspace are orthogonal to each other. A different approach, presented by Wang et al., makes use of some auxiliary antenna elements [16]. In [17, 18,21] a calibration algorithm is presented, which utilizes the ephemeris from each satellite. A fairly new proposed method exploits the spatial sparsity of the incident signals using the Sparse Bayesian 


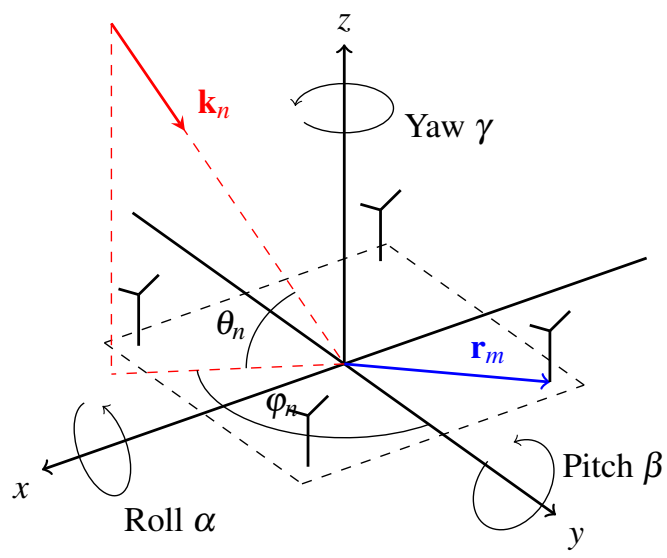

Figure 1: Antenna array configuration

Learning method $[19,20]$. However, it turns out that the techniques mentioned above still have some disadvantages. In the case that the ephemeris from each satellite are utilized, the attitude of the antenna array needs to be estimated. However, in [21] it is simply assumed to be known. Moreover, some proposed methods either assume the gain and phase mismatches (approximately) to be known [11,14-17,20] or neglect the effect of mutual coupling [10,18]. Others rely on an extensive set of antenna elements that is usually not practical for GNSS applications or assume that the distance between some antenna elements is large enough, so that mutual coupling can be neglected $[13,14,16,19,20]$. Some also suppose that the mutual coupling matrix has special kind of structure $[11,13-17,19,20]$, that is, however, not the case for crosstalk-effects.

This paper proposes a novel algorithm, which compensates for gain and phase mismatches as well as crosstalk-effects. Moreover, it will also estimate the attitude of the antenna array. It does not make any assumption about the array geometry and it is also feasible for a small number of antenna elements. Similar to $[17,18]$ the proposed method is based on the ephemeris from each satellite that is sent along with the navigation data. From the ephemeris an expected steering vector is derived, which is compared with the measured steering vector to estimate the unknown parameters. For this to work, the attitude of the antenna array is a mandatory requirement as the ephemeris are given in the East-North-Up (ENU) coordinate system, whereas the measured steering vectors correspond to the local coordinate system. Therefore, a maximum-likelihood approach is presented to estimate the gain and phase mismatches, crosstalk-effects as well as the attitude of the antenna array.

\section{SYSTEM MODEL}

Consider an $M$-element antenna array with an arbitrary configuration (see Figure 1). For $N$ impinging GNSS signals onto the antenna array the wave vector $\mathbf{k}_{n}$ is defined as

$$
\mathbf{k}_{n}=-\frac{2 \pi}{\lambda}\left(\begin{array}{c}
\cos \left(\theta_{n}\right) \sin \left(\varphi_{n}\right) \\
\cos \left(\theta_{n}\right) \cos \left(\varphi_{n}\right) \\
\sin \left(\theta_{n}\right)
\end{array}\right), n=1, \cdots, N,
$$

where $\varphi_{n}$ and $\theta_{n}$ are the azimuth and elevation angle of the $n$-th impinging signal respectively and $\lambda$ is the wavelength. With the antenna position vector $\mathbf{r}_{m}$ the steering vector is determined by

$$
\mathbf{a}\left(\varphi_{n}, \theta_{n}\right)=\left(\begin{array}{c}
e^{-\mathrm{j} \mathbf{k}_{n}^{\mathrm{T}} \mathbf{r}_{1}} \\
\vdots \\
e^{-\mathrm{j} \mathbf{k}_{n}^{\mathrm{T}} \mathbf{r}_{M}}
\end{array}\right) .
$$




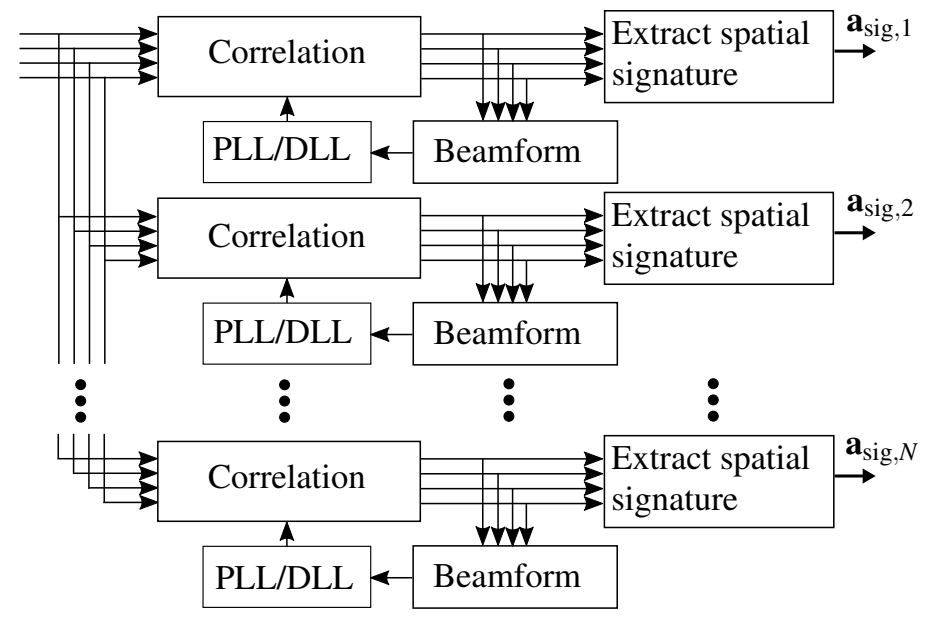

Figure 2: Receiver architecture

Assuming no antenna array uncertainties a perfect array output would yield

$$
\mathbf{x}(t)=\sum_{n=1}^{N} \mathbf{a}\left(\varphi_{n}, \theta_{n}\right) s_{n}(t)+\mathbf{n}(t),
$$

where $\mathbf{n}(t)$ represents Gaussian white noise of variance $\sigma_{\mathrm{n}}^{2}$ and $s_{n}(t)$ the GNSS signal containing the PRN-Code and carrier. However, as mentioned above there are a number of array uncertainties, which have to be compensated. The gain and phase mismatches introduced by the individual direction dependent radiation characteristics of each antenna element are termed $\mathbf{g}\left(\varphi_{n}, \theta_{n}\right) \in \mathbb{C}^{M \times 1}$. The mutual coupling is modeled by a symmetric matrix $\mathbf{M} \in \mathbb{C}^{M \times M}$ with the diagonal entries being $[\mathbf{M}]_{m, m}=1$. Besides that, the mutual coupling matrix in general has no special structure. However, in the case of uniform linear arrays or uniform circular arrays a Toeplitz matrix or a circulant Toeplitz matrix will provide an excellent model respectively [11]. The rationale behind this model is the fact that the mutual coupling coefficients are inversely proportional to the distance between the antenna elements. The time varying contribution is modeled by $\mathbf{C} \in \mathbb{C}^{M \times M}$, where the diagonal entries hold the gain and phase mismatches introduced by active elements like amplifiers, downconverters as well as various filters and unequal cable lengths. The non diagonal entries hold the crosstalk uncertainties. This matrix is usually not symmetric as it holds the multiplication of a number of consecutive gain and phase mismatches as well as crosstalk-effects. Additionally it can hold uncertainties introduced by spatial filters, that are used to mitigate interferences. The antenna array output finally yields

$$
\mathbf{x}(t)=\sum_{n=1}^{N} \mathbf{C} \mathbf{M} \mathbf{g}\left(\varphi_{n}, \theta_{n}\right) \odot \mathbf{a}\left(\varphi_{n}, \theta_{n}\right) s_{n}(t)+\mathbf{n}(t)
$$

where $\odot$ denotes the Hadamard product. In theory $\mathbf{C}$ and $\mathbf{M}$ can be reduced to one matrix, but are separated here for the sake of clarification of their origin and their time dependence. The matrix $\mathbf{C}$ contains the time-varying contribution, whereas $\mathbf{M}$ and $\mathbf{g}\left(\varphi_{n}, \theta_{n}\right)$ are known to be stable over time. Therefore, the latter can be measured in an anechoic chamber during manufacturing process and saved in a look up table. Usually it saved in combination with the steering vector, that forms the measured steering vector $\widetilde{\mathbf{a}}\left(\varphi_{n}, \theta_{n}\right)=\mathbf{M} \mathbf{g}\left(\varphi_{n}, \theta_{n}\right) \odot \mathbf{a}\left(\varphi_{n}, \theta_{n}\right)$. The basic GNSS receiver architecture is given in Figure 2. Each multi-antenna correlator is controlled by one Phase Locked Loop (PLL) and one Delay Locked Loop (DLL) using the beamformed output. Thus, the phase relationship among the different antenna channels is preserved and the correlator output yields

$$
\mathbf{y}_{n}(t)=\mathbf{C} \widetilde{\mathbf{a}}\left(\varphi_{n}, \theta_{n}\right) p_{n}(t)+\breve{\mathbf{n}}(t),
$$

where $p_{n}(t)$ is the amplitude and phase of the $n$-th GNSS signal, $\breve{\mathbf{n}}(t)$ Gaussian white noise with variance $\frac{1}{T} \sigma_{\mathrm{n}}^{2}$ and $T$ the integration time. 


\section{Spatial Signal Signature}

From the correlator outputs in (5) a spatial signal signature

$$
\mathbf{a}_{\mathrm{sig}, n}=\frac{1}{L} \sum_{l=0}^{L-1} \mathbf{y}_{n}(l T)
$$

can be extracted by averaging over $L$ correlator epochs. Note that the data signal needs to be stripped off before taking the average. Here it is assumed that $\mathbf{y}_{n}(t)$ does not contain any data. The spatial signal signature will be of significant importance in the further course of this paper. Note that the spatial signal signature still retains the phase relationships between the signals from the different antennas.

\section{Ephemeris}

Each satellite sends its ephemeris along with the navigation data. As long as the user position is roughly known, an expected DOA can be derived from the ephemeris. Small errors in the estimated user position will not have great impact on the expected DOA due to the long distance between the user and the satellites. The ephemeris are given in the ENU coordinate frame, whereas the estimated DOAs found by direction finding algorithms are given in the local coordinate frame of the antenna array. To compare both one has to be rotated by the antenna attitude onto the other. For a perfectly calibrated antenna array the problem can be mathematically expressed as

$$
\widehat{\mathbf{d}}_{n}=\mathbf{T}(\gamma, \beta, \alpha) \mathbf{d}_{\exp , n}+\mathbf{n}_{\mathrm{T}, n},
$$

where $\widehat{\mathbf{d}}_{n}$ and $\mathbf{d}_{\exp , n}$ are unit vectors representing the estimated DOA from the direction finding algorithm and the expected DOA from the ephemeris for the $n$-th imping signal respectively and $\mathbf{n}_{\mathrm{T}, n}$ is additive noise. $\mathbf{T}$ is the rotation matrix, that is determined by the unknown antenna attitude angles yaw $\gamma$, pitch $\beta$ and roll $\alpha$ (see Figure 1) by

$$
\mathbf{T}(\gamma, \beta, \alpha)=\mathbf{T}_{\alpha} \mathbf{T}_{\beta} \mathbf{T}_{\gamma}
$$

with

$$
\mathbf{T}_{\gamma}=\left(\begin{array}{ccc}
\cos \gamma & -\sin \gamma & 0 \\
\sin \gamma & \cos \gamma & 0 \\
0 & 0 & 1
\end{array}\right), \quad \mathbf{T}_{\beta}=\left(\begin{array}{ccc}
\cos \beta & 0 & \sin \beta \\
0 & 1 & 0 \\
-\sin \beta & 0 & \cos \beta
\end{array}\right), \quad \mathbf{T}_{\alpha}=\left(\begin{array}{ccc}
1 & 0 & 0 \\
0 & \cos \alpha & -\sin \alpha \\
0 & \sin \alpha & \cos \alpha
\end{array}\right)
$$

\section{PROPOSED METHOD}

In the following a novel algorithm to estimate the gain and phase mismatches, crosstalk-effects and the attitude of the antenna array will be presented. Due to the fact that the PLL and DLL are driven by the beamformed signal, there is a constant phase $p_{n}$ being added to the correlator outputs. Unfortunately this phase does not cancel out when taking the average to obtain the spatial signature. Therefore, this phase has to be estimated as well. The basic concept of the proposed algorithm is to use the ephemeris from the satellites in view to derive an expected steering vector, that is compared with the corresponding estimated spatial signature.

From equations (2) and (7) an expected steering vector for each impinging GNSS signal can be formulated as

$$
\mathbf{a}_{\exp , n}(\boldsymbol{\alpha})=\left(\begin{array}{c}
e^{\mathrm{j} \frac{2 \pi}{\lambda}\left(\mathbf{T}(\gamma, \beta, \alpha) \mathbf{d}_{\mathrm{exp}, n}\right)^{\mathrm{T}} \mathbf{r}_{1}} \\
\vdots \\
e^{\mathrm{j} \frac{2 \pi}{\lambda}\left(\mathbf{T}(\gamma, \beta, \alpha) \mathbf{d}_{\mathrm{exp}, n}\right)^{\mathrm{T}} \mathbf{r}_{M}}
\end{array}\right) \text {, with } \boldsymbol{\alpha}=\left(\begin{array}{c}
\alpha \\
\beta \\
\gamma
\end{array}\right) .
$$

From equation (5) a cost function can be defined to estimate the unknown coefficients:

$$
\begin{aligned}
J(\boldsymbol{\alpha}, \mathbf{C}, \mathbf{s}) & =\sum_{n=1}^{N}\left\|\mathbf{a}_{\mathrm{sig}, n}-\mathbf{C} \mathbf{M} \mathbf{g}\left(\varphi_{n}, \theta_{n}\right) \odot \mathbf{a}_{\exp , n}(\boldsymbol{\alpha}) p_{n}\right\|^{2}=\sum_{n=1}^{N}\left\|\mathbf{f}_{n}(\boldsymbol{\alpha}, \mathbf{C}, \mathbf{p})\right\|^{2} \\
& \text { with } \mathbf{f}_{n}(\boldsymbol{\alpha}, \mathbf{C}, \mathbf{s})=\mathbf{a}_{\mathrm{sig}, n}-\mathbf{C} \mathbf{M g}\left(\varphi_{n}, \theta_{n}\right) \odot \mathbf{a}_{\exp , n}(\boldsymbol{\alpha}) p_{n},
\end{aligned}
$$




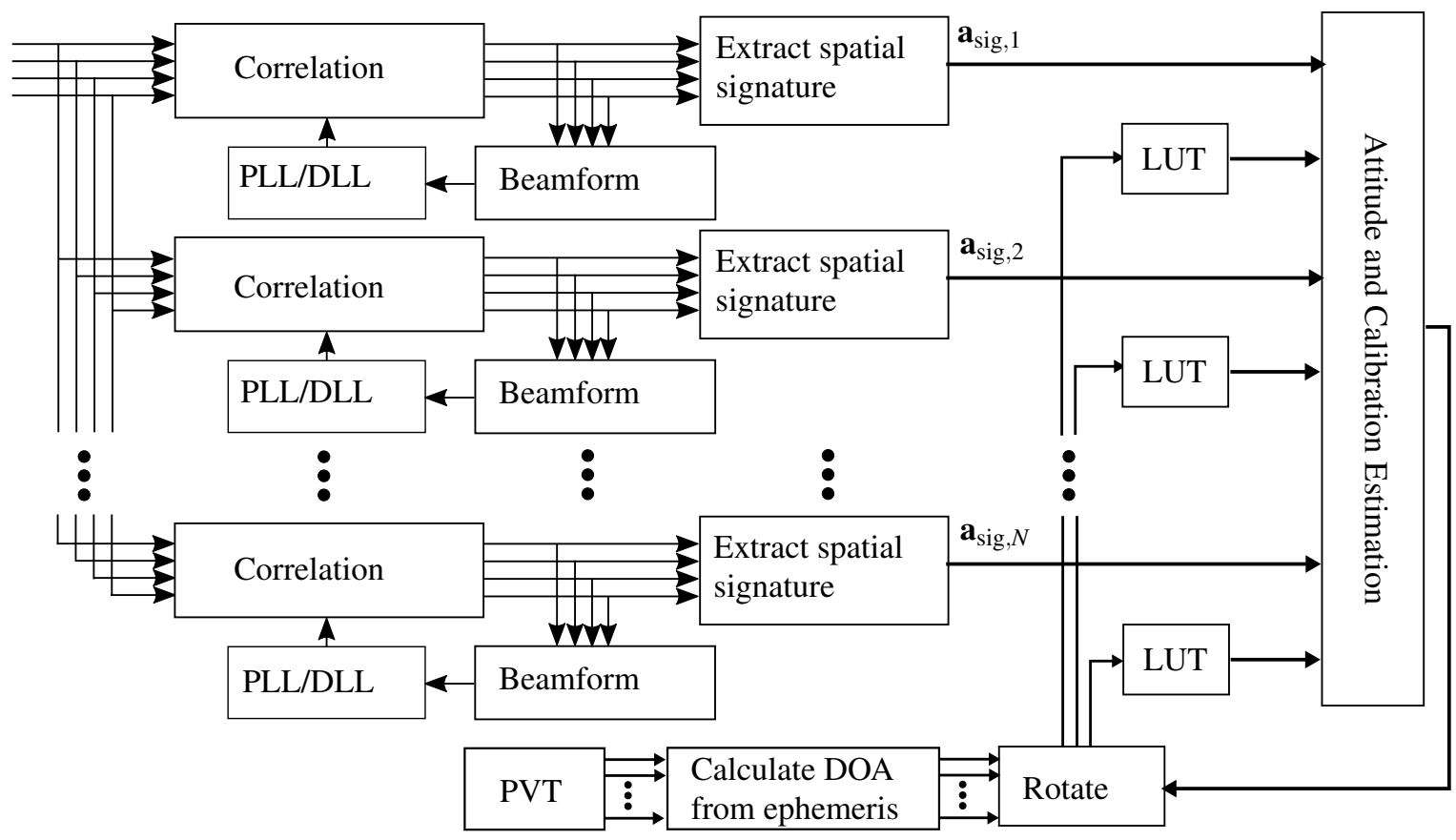

Figure 3: Receiver architecture to jointly estimate the antenna array attitude and calibration

where $\mathbf{p}$ contains the unknown amplitude and phase of the GNSS signals $\mathbf{p}=\left(\begin{array}{lll}p_{1} & \cdots & p_{N}\end{array}\right)$. The unknown matrix $\mathbf{C}$ is normed to its last element to avoid ambiguity with the matrix $\mathbf{p}$, so that $[\mathbf{C}]_{M, M}=1$ holds. Since $\mathbf{C}$, the antenna attitude $\boldsymbol{\alpha}$ and $\mathbf{p}$ contain $2\left(M^{2}-1\right), 3$ and $2 N$ real unknowns respectively and the number of independent measurements is $2 M N$, a necessary condition for (11) to have solutions for $\mathbf{C}, \boldsymbol{\alpha}$ and $\mathbf{p}$ is that

$$
\begin{aligned}
2 M N & \geq 2\left(M^{2}-1\right)+3+2 N \\
\Rightarrow N & \geq \frac{M^{2}+0.5}{M-1}=M+1+\frac{1.5}{M-1} .
\end{aligned}
$$

That means for an antenna array with 4 elements at least 6 GNSS signals from distinct DOAs are needed.

The cost function (11) can be minimized iteratively using the Levenberg-Marquardt algorithm. With $\mathrm{k}$ being the k-th iteration and $\mathbf{c}=[\operatorname{vec}(\mathbf{C})]_{1: M^{2}-1}$ the Jacobian matrix is derived as

$$
\mathbf{J}_{k}=\left(\begin{array}{ccc}
\frac{\delta \mathbf{f}_{1}^{(k)}}{\delta \boldsymbol{\alpha}} & \frac{\delta \mathbf{f}_{1}^{(k)}}{\delta \mathbf{c}} & \frac{\delta \mathbf{f}_{1}^{(k)}}{\delta \mathbf{p}} \\
\vdots & \vdots & \vdots \\
\frac{\delta \mathbf{f}_{N}^{(k)}}{\delta \boldsymbol{\alpha}} & \frac{\delta \mathbf{f}_{N}^{(k)}}{\delta \mathbf{c}} & \frac{\delta \mathbf{f}_{N}^{(k)}}{\delta \mathbf{p}}
\end{array}\right)
$$

The various derivatives are given in the appendix. To derive $\mathbf{f}_{n}^{(k)}$ the mutual coupling matrix in combination with the antenna radiation characteristic and the steering vector $\mathbf{M} \mathbf{g}\left(\varphi_{n}, \theta_{n}\right) \odot \mathbf{a}_{\exp , n}(\boldsymbol{\alpha})$ can be read from the LUT by first rotating the expected DOA unit vector using the current rotation matrix $\left(x_{n}^{(k)}, y_{n}^{(k)}, z_{n}^{(k)}\right)^{\mathrm{T}}=\mathbf{T}\left(\widehat{\boldsymbol{\alpha}}^{(k)}\right) \mathbf{d}_{\mathrm{exp}, n}$, secondly calculating the azimuth and elevation using $\varphi_{\mathrm{rot}, n}^{(k)}=\arctan 2\left(\frac{x_{n}^{(k)}}{y_{n}^{(k)}}\right)$ and $\theta_{\mathrm{rot}, n}^{(k)}=\arcsin \left(z_{n}^{(k)}\right)$ and subsequently reading the values from the LUT with $\widetilde{\mathbf{a}}_{\mathrm{exp}, n}^{(k)}=\widetilde{\mathbf{a}}\left(\varphi_{\mathrm{rot}, n}^{(k)}, \theta_{\mathrm{rot}, n}^{(k)}\right)$. The 


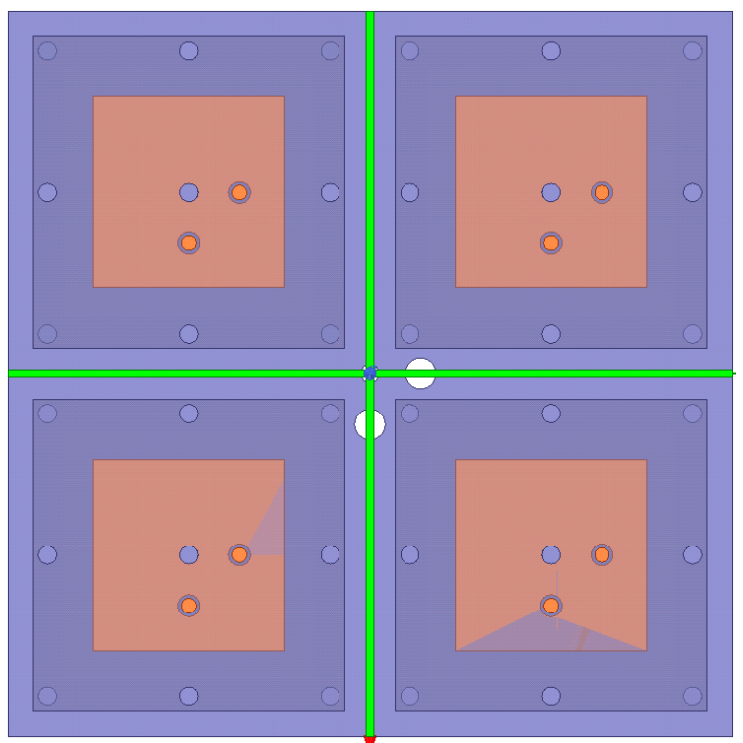

(a) Miniaturized antenna array used for simulations

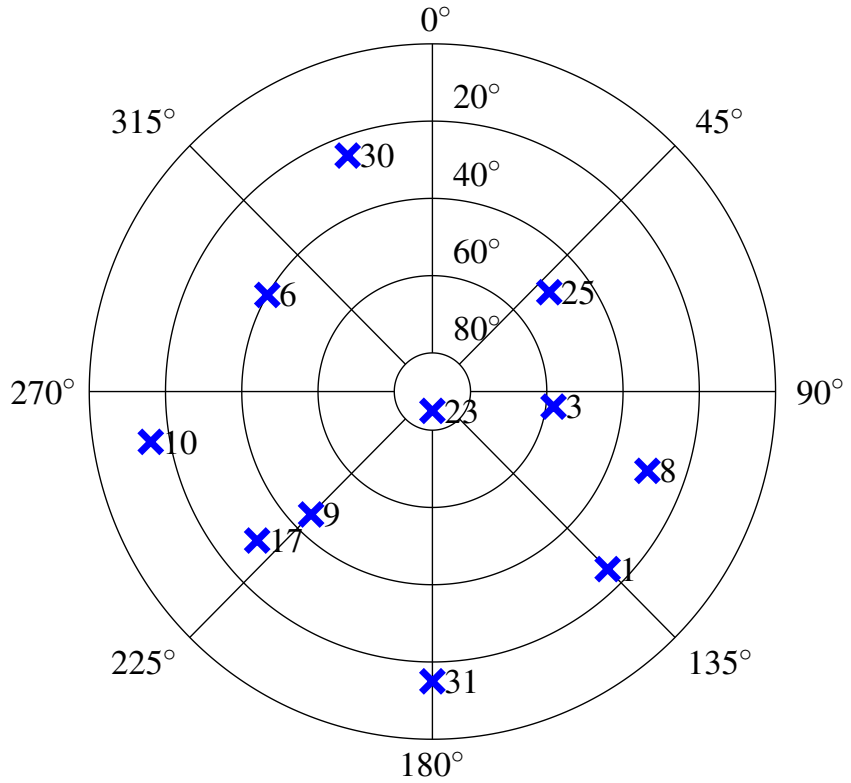

(b) Simulated satellite constellation

Figure 4: Simulation setup

updates for the unknown coefficients are then calculated by

$$
\left(\begin{array}{c}
\Delta \widehat{\boldsymbol{\alpha}}^{(k)} \\
\Delta \widehat{\mathbf{c}}^{(k)} \\
\Delta \widehat{\mathbf{p}}^{(k)}
\end{array}\right)=\left(\mathbf{J}^{(k) \mathrm{T}} \mathbf{J}^{(k)}+\mu \mathbf{I}\right)^{-1} \mathbf{J}^{(k) \mathrm{T}}\left(\begin{array}{c}
\mathbf{f}_{1}^{(k)} \\
\vdots \\
\mathbf{f}_{N}^{(k)}
\end{array}\right) .
$$

The Levenberg-Marquardt parameter $\mu$ has to be chosen in such a way that $\sum_{n=1}^{N}\left\|\mathbf{f}_{n}^{(k)}\right\|^{2}>\sum_{n=1}^{N}\left\|\mathbf{f}_{n}^{(k+1)}\right\|^{2}$. When the scalar $\mu$ is zero, this optimization algorithm is identical to the Gauss-Newton algorithm. When $\mu$ is large, this becomes gradient descent with a small step size. Gauss-Newton's method is faster and more accurate near the cost functions minimum, so the aim is to shift toward Gauss-Newton's method as quickly as possible. In practice heuristic methods are used to drive $\mu$ [22]. For the next iteration the addressed unknowns are updated by the previously derived updates:

$$
\left(\begin{array}{c}
\widehat{\boldsymbol{\alpha}}^{(k+1)} \\
\widehat{\mathbf{c}}^{(k+1)} \\
\widehat{\mathbf{p}}^{(k+1)}
\end{array}\right)=\left(\begin{array}{c}
\widehat{\boldsymbol{\alpha}}^{(k)} \\
\widehat{\mathbf{c}}^{(k)} \\
\widehat{\mathbf{p}}^{(k)}
\end{array}\right)+\left(\begin{array}{c}
\operatorname{Re}\left\{\Delta \widehat{\boldsymbol{\alpha}}^{(k)}\right\} \\
\Delta \widehat{\mathbf{c}}^{(k)} \\
\Delta \widehat{\mathbf{p}}^{(k)}
\end{array}\right),
$$

where $\operatorname{Re}\{$.$\} denotes taking the real part. The real part is taken for the antenna attitude as the angles yaw \gamma$, pitch $\beta$ and roll $\alpha$ are real values. The iteration can be terminated when the norm of the updates in (16) is below a predefined threshold. Subsequently, the spatial signature $\mathbf{a}_{\mathrm{sig}, n}$ can be calibrated by

$$
\mathbf{a}_{\mathrm{cal}, n}=\widehat{\mathbf{C}}^{-1} \mathbf{a}_{\mathrm{sig}, n}
$$

The corresponding receiver architecture is given in Figure 3. The algorithm can be summarized by the following steps:

1. Initialize the unknown parameters $\widehat{\mathbf{C}}^{(0)}=\mathbf{I}, \widehat{\mathbf{p}}^{(0)}=(1, \cdots, 1)^{\mathrm{T}}$ and $\widehat{\boldsymbol{\alpha}}^{(0)}=(0,0,0)$.

2. Rotate the DOA from the ephemeris onto the local coordinate frame using the current rotation matrix $\mathbf{T}\left(\widehat{\boldsymbol{\alpha}}^{(k)}\right)$. 


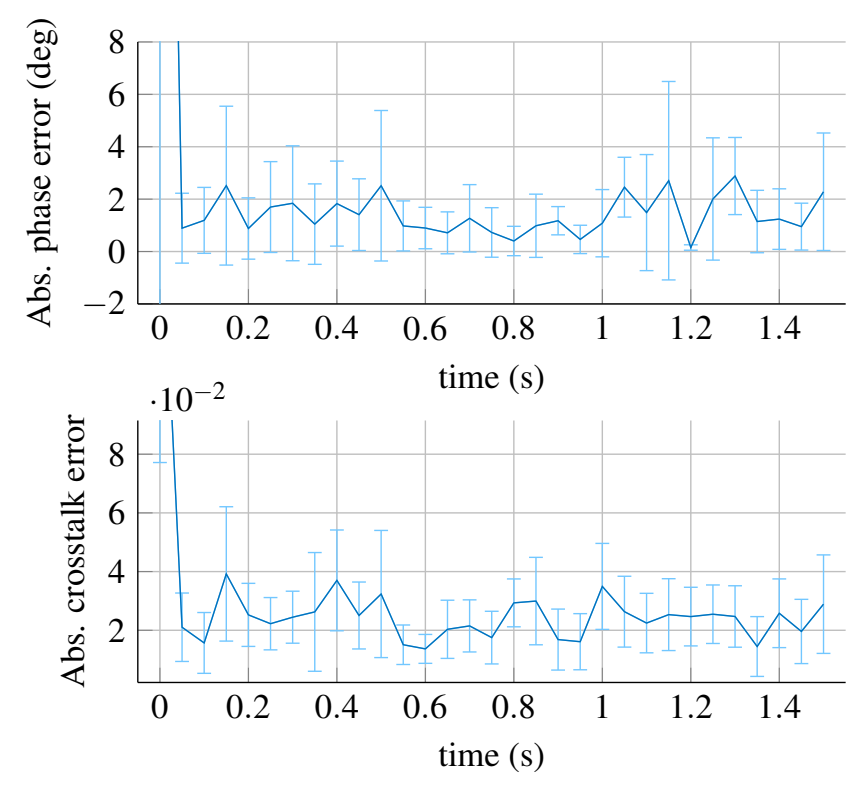

(a) Calibration error
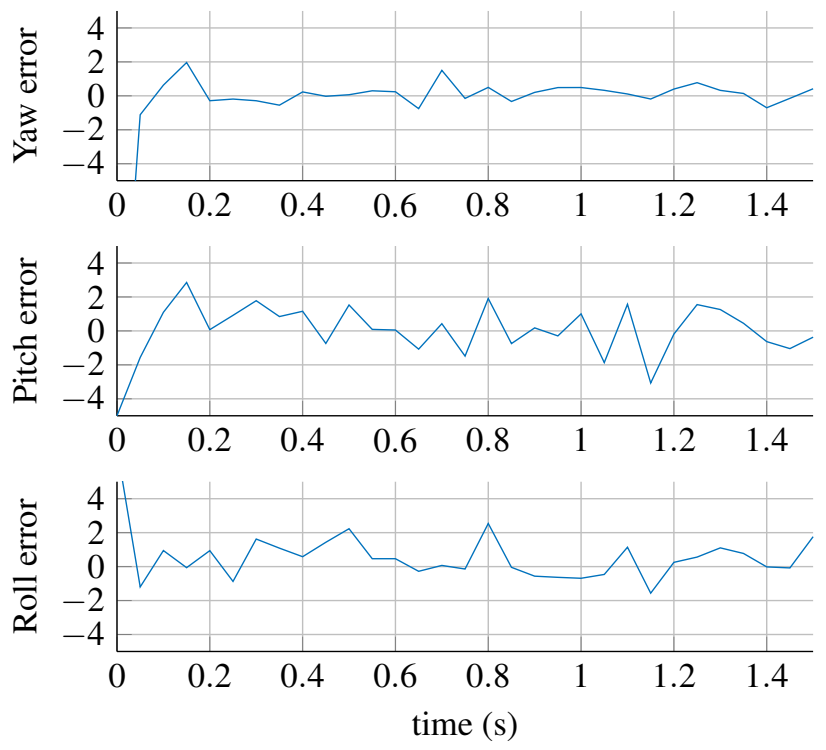

(b) Attitude error

Figure 5: Simulation results

3. Look up the corresponding steering vector from the LUT to get the expected steering vector in combination with the antenna characteristic $\widetilde{\mathbf{a}}_{\mathrm{exp}, n}^{(k)}$

4. Update the unknown parameters using the Levenberg-Marquardt method.

5. Return to step 2 if the norm of the update is greater than a predefined threshold.

\section{SIMULATION RESULTS}

To test the proposed algorithm a miniaturized 4-element uniform rectangular array (URA) with metallic fences according to [8] was simulated (see Figure 4a). The green lines indicate the metallic fences and the orange squares the antenna patches. The mutual distance between the nearby antenna elements is $50 \mathrm{~mm}$. The total size of the antenna array is $100 \mathrm{~mm} \times 100 \mathrm{~mm}$. The antenna supports two circular polarized antenna outputs. For the purpose of this paper only the right hand circular polarized (RHCP) output is used. During the simulation of the array manifold the antenna elements were arranged as follows:

$$
\left(\begin{array}{llll}
\mathbf{r}_{1} & \mathbf{r}_{2} & \mathbf{r}_{3} & \mathbf{r}_{4}
\end{array}\right)=\frac{0.05}{2}\left(\begin{array}{cccc}
-1 & 1 & 1 & -1 \\
1 & 1 & -1 & -1 \\
0 & 0 & 0 & 0
\end{array}\right) .
$$

The simulated output $\widetilde{\mathbf{a}}\left(\varphi_{i}, \theta_{i}\right)$ is saved in a look-up-table with a resolution of $1^{\circ}$ for $0^{\circ} \leq \varphi_{i}<360^{\circ}$ and $0^{\circ} \leq \theta_{i} \leq 90^{\circ}$. The values in between are linearly interpolated when needed. On top of the simulated array manifold a gain and phase offset among the antenna channels and some crosstalk-effects are added. For a first test the standard deviation between the phase offsets is set to $30^{\circ}$, the gain is set to 1 and the crosstalk-effects are set randomly with a magnitude of $10 \mathrm{~dB}$ below the gain. The simulated satellite constellation is given in Figure $4 \mathrm{~b}$. The attitude angles yaw, pitch and roll are set to $\gamma_{\mathrm{sim}}=20^{\circ}, \beta_{\mathrm{sim}}=5^{\circ}$ and $\alpha_{\text {sim }}=-7^{\circ}$ respectively. The signal that drives the PLL / DLL is beamformed by an eigenbeamformer [1]. The results for the calibration and attitude estimation are given in Figure 5. The upper figure in Figure 5a shows the mean and standard deviation of the phase angle

$$
\mathbf{e}_{\text {phase }}=\left|\arg \left(\left[\operatorname{diag}\left(\widehat{\mathbf{C}}^{*}\right)\right]_{1: M-1} \odot\left[\operatorname{diag}\left(\frac{\mathbf{C}_{\text {sim }}}{\left[\mathbf{C}_{\text {sim }}\right]_{M, M}}\right)\right]_{1: M-1}\right)\right|
$$




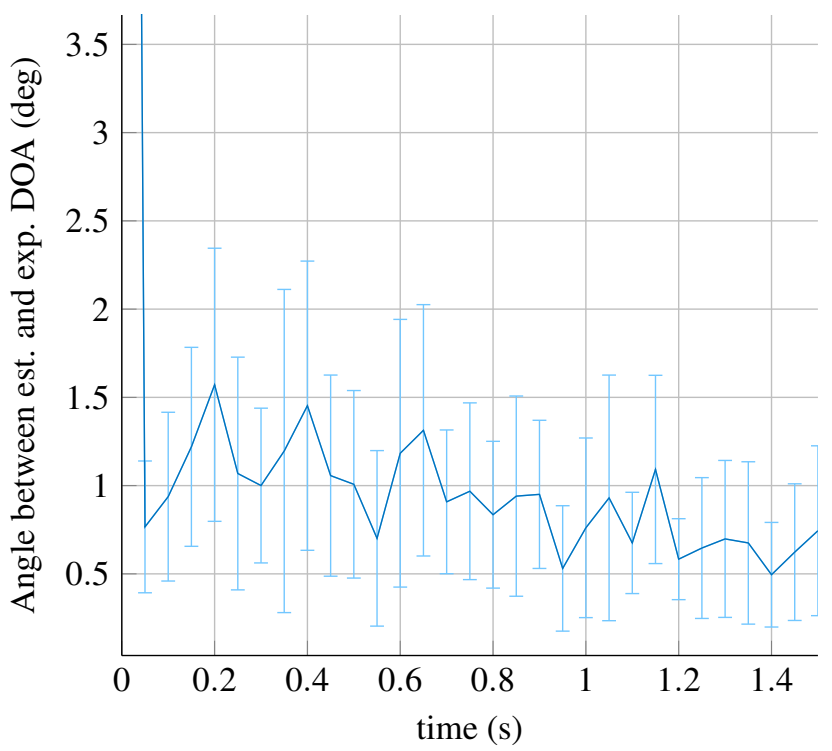

(a) Angle between est. and exp. DOA

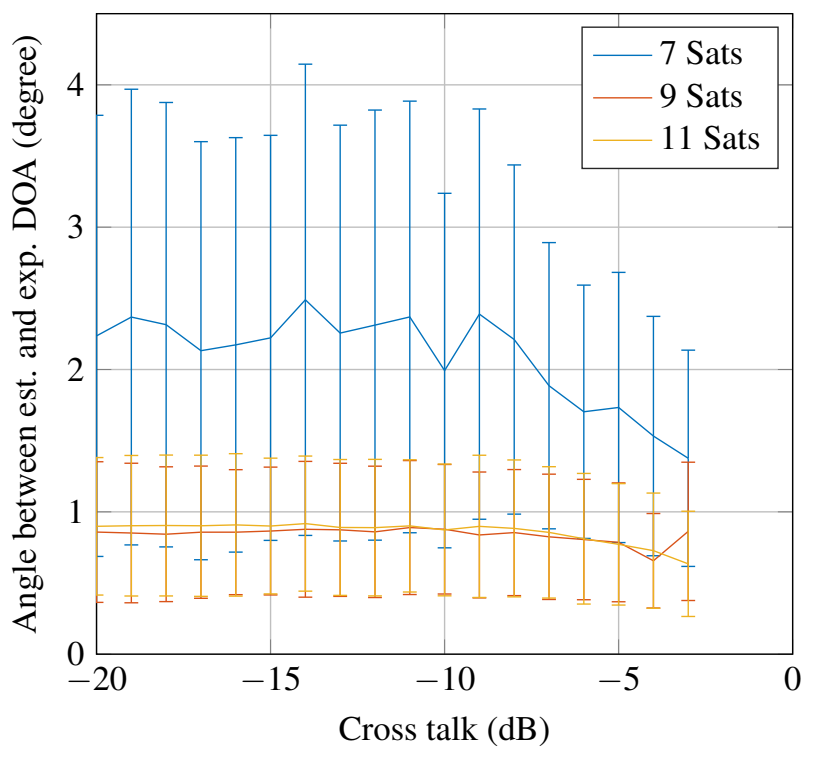

(b) Angle between est. and exp. DOA for different crosstalk amplitudes and number of satellites

Figure 6: Simulation results

between the estimated and the simulated gain and phase mismatches, where $\mathbf{C}_{\text {sim }}$ contains the simulated gain and phase mismatches as well as the crosstalk-effects, $\arg ($.$) returns the phase of a complex number in degree from -180^{\circ}$ to $180^{\circ}, \operatorname{diag}(\mathbf{A}) \operatorname{returns}$ the diagonal elements of $\mathbf{A}$ and $\mathbf{A}^{*}$ is the conjugate of $\mathbf{A}$. Note that because $\widehat{\mathbf{C}}$ is normed to its last element, $\mathbf{C}_{\text {sim }}$ has to be normed to its last element as well. Without any calibration the error starts with a mean value of $44.17^{\circ}$ and standard deviation of $62.75^{\circ}$. As soon as the algorithm kicks in the phase error is lowered toward a mean value of $1.38^{\circ}$ and a standard deviation of $1.41^{\circ}$. The lower figure in Figure 5a shows the mean and standard deviation of the crosstalk estimation error

$$
\mathbf{e}_{\text {crosstalk }}=\sqrt{\text { offdiag }\left(\widehat{\mathbf{C}}^{*}\right) \odot \text { offdiag }\left(\frac{\mathbf{C}_{\text {sim }}}{\left[\mathbf{C}_{\text {sim }}\right]_{M, M}}\right)},
$$

where offdiag $(\mathbf{A})$ denotes taking the off-diagonal elements of matrix $\mathbf{A}$ and $\sqrt{ }$. taking the element-wise square root. Without any calibration the error starts with a mean value of 0.18 and standard deviation of 0.10 . As soon as the algorithm kicks in the absolute crosstalk error is lowered toward a mean value of 0.02 and a standard deviation of 0.01 .

The estimation errors for the attitude of the antenna array

$$
\mathbf{e}_{\text {attitude }}=\widehat{\boldsymbol{\alpha}}-\boldsymbol{\alpha}_{\text {sim }}
$$

are given in Figure $5 b$, where $\boldsymbol{\alpha}_{\text {sim }}$ contains the simulated attitude angles yaw, pitch and roll. After the first estimate the yaw error drops down to $0.31^{\circ}$, the pitch error drops down to $0.84^{\circ}$ and the roll error drops down to $1.08^{\circ}$.

Another critical measure for the performance of the proposed algorithm is how well an estimated DOA fits to the corresponding expected DOA from the ephemeris. If both match perfectly, deterministic spatial nulls and / or enhancements can be placed to either mitigate spoofers / jammers or enhance the signal to noise ratio (SNR) of GNSS signals. The estimated DOA is determined by means of standard MUSIC algorithm:

$$
\widehat{\varphi}_{n}, \widehat{\theta}_{n}=\arg \max _{\varphi, \theta} \frac{\left|\mathbf{a}_{\mathrm{sig}, n}^{\mathrm{H}} \widehat{\mathbf{C}} \widetilde{\mathbf{a}}(\varphi, \theta)\right|^{2}}{(\widehat{\mathbf{C}} \widetilde{\mathbf{a}}(\varphi, \theta))^{\mathrm{H}} \widehat{\mathbf{C}} \widetilde{\mathbf{a}}(\varphi, \theta)},
$$


where there the measured array manifold $\widetilde{\mathbf{a}}(\varphi, \theta)$ is corrected by the estimated gain and phase mismatches and crosstalk-effects. Subsequently, the estimated DOA in the Cartesian domain is given by

$$
\widehat{\mathbf{d}}_{n}=\left(\begin{array}{c}
\cos \left(\widehat{\theta}_{n}\right) \sin \left(\widehat{\varphi}_{n}\right) \\
\cos \left(\widehat{\theta}_{n}\right) \cos \left(\widehat{\varphi}_{n}\right) \\
\sin \left(\widehat{\theta}_{n}\right)
\end{array}\right) .
$$

Finally, the angle between the estimated and expected DOA yields

$$
\mathbf{e}_{\text {angle }}=\arccos \left(\widehat{\mathbf{d}}_{n}^{\mathrm{T}}\left(\mathbf{T}(\widehat{\boldsymbol{\alpha}}) \mathbf{d}_{\mathrm{exp}, n}\right)\right) .
$$

The mean and standard deviation of $\mathbf{e}_{\text {angle }}$ is given in Figure 6a. Before any calibration the mean angle between the estimated and expected DOAs is $21.09^{\circ}$ and its standard deviation is $13.93^{\circ}$. After calibration the mean angle drops down to $0.92^{\circ}$ and its standard deviation drops down to $0.27^{\circ}$.

Another simulation is carried out to test the performance of the proposed algorithm based on different crosstalk amplitudes and number of satellites. Figure $6 \mathrm{~b}$ shows the mean and standard deviation of the angle between the estimated and expected DOAs averaged over 60 snapshots. The satellite constellation is depicted from Figure 4b, where 7 Sats depicts the subset $(1,3,6,8,9,10$, 17), 9 Sats depicts the subset $(1,3,6,8,9,10,17,23,25)$ and 11 Sats includes all satellites. The performance with only 7 satellites in view is already very well with an error of only around $2^{\circ}$. When the number of satellites is increased to 9 or more the angle between the estimated and expected DOA drops below $1^{\circ}$.

\section{SUMMARY}

Antenna array attitude and calibration estimation is an important component, if deterministic beamforming, spoofing detection and mitigation or jammer localization is of interest. In this paper a novel algorithm has been presented to estimate the attitude of the antenna array and to calibrate the signal phase offsets among the different antenna channels as well as crosstalk-effects without using any additional hardware like an inertial measurement unit or a calibration signal, which is fed back into the antenna elements. The novel algorithm provides a very good match between estimated DOAs and the expected DOAs obtained by the ephemeris. That means the ephemeris data can be used to do deterministic spatial processing to either enhance the LOS signal, or detect and mitigate any unwanted signals like jammers or spoofers. The proposed method has been tested in simulations using a miniaturized antenna array. It has yet to be verified with recorded data.

\section{ACKNOWLEDGMENTS}

Parts of the research leading to the results reported in this paper have been funded within the project PRS Protect by the German Aerospace Center (DLR) on behalf of the German Federal Ministry of Economics and Technology under grant no. 50 NP 1604. This support is greatly acknowledged.

\section{APPENDIX}

The derivatives for the Jakobi-Matrix defined in (15) are derived as

$$
\frac{\delta \mathbf{f}_{n}^{(k)}}{\delta \boldsymbol{\alpha}}=\left(\begin{array}{lll}
\frac{\delta \mathbf{f}_{n}^{(k)}}{\delta \alpha} & \frac{\delta \mathbf{f}_{n}^{(k)}}{\delta \beta} & \frac{\delta \mathbf{f}_{n}^{(k)}}{\delta \gamma}
\end{array}\right), \quad \frac{\delta \mathbf{f}_{n}^{(k)}}{\delta \mathbf{c}}=\left(\begin{array}{ccc}
\frac{\delta \mathbf{f}_{n}^{(k)}}{\delta c_{1}} & \cdots & \frac{\delta \mathbf{f}_{n}^{(k)}}{\delta c_{M^{2}-1}}
\end{array}\right), \quad \frac{\delta \mathbf{f}_{n}^{(k)}}{\delta \mathbf{p}}=\left(\begin{array}{lll}
\frac{\delta \mathbf{f}_{n}^{(k)}}{\delta p_{1}} & \cdots & \frac{\delta \mathbf{f}_{n}^{(k)}}{\delta p_{N}}
\end{array}\right)
$$


where

$$
\begin{aligned}
& \frac{\delta \mathbf{f}_{n}^{(k)}}{\delta \alpha}=-\widehat{\mathbf{C}}^{(k)}\left[\begin{array}{c}
\widetilde{\mathbf{a}}_{\mathrm{exp}, n}^{(k)} \odot\left(\begin{array}{c}
\frac{2 \pi}{\lambda}\left(\frac{\delta \mathbf{T}_{\widehat{\alpha}^{(k)}}}{\delta \alpha} \mathbf{T}_{\widehat{\boldsymbol{\beta}}^{(k)}} \mathbf{T}_{\widehat{\gamma}^{(k)}} \mathbf{d}_{\mathrm{exp}, n}\right)^{\mathrm{T}} \mathbf{r}_{1} \\
\vdots \\
\frac{2 \pi}{\lambda}\left(\frac{\delta \mathbf{T}_{\widehat{\alpha}^{(k)}}}{\delta \alpha} \mathbf{T}_{\widehat{\beta}^{(k)}} \mathbf{T}_{\widehat{\gamma}^{(k)}} \mathbf{d}_{\mathrm{exp}, n}\right)^{\mathrm{T}} \mathbf{r}_{M}
\end{array}\right]+\widehat{\mathbf{M}}^{(k)} \frac{\delta \mathbf{g}}{\delta \alpha} \odot \mathbf{a}\left(\varphi_{\mathrm{rot}, n}^{(k)}, \theta_{\mathrm{rot}, n}^{(k)}\right)
\end{array}\right] \widehat{p}_{n}^{(k)}, \\
& \frac{\delta \mathbf{f}_{n}^{(k)}}{\delta \beta}=-\widehat{\mathbf{C}}^{(k)}\left[\widetilde{\mathbf{a}}_{\mathrm{exp}, n}^{(k)} \odot\left(\begin{array}{c}
\frac{2 \pi}{\lambda}\left(\mathbf{T}_{\widehat{\alpha}^{(k)}} \frac{\delta \mathbf{T}_{\widehat{\beta}^{(k)}}}{\delta \beta} \mathbf{T}_{\widehat{\gamma}^{(k)}} \mathbf{d}_{\mathrm{exp}, n}\right)^{\mathrm{T}} \mathbf{r}_{1} \\
\vdots \\
\frac{2 \pi}{\lambda}\left(\mathbf{T}_{\widehat{\alpha}^{(k)}} \frac{\delta \mathbf{T}_{\widehat{\beta}^{(k)}}^{\delta \beta}}{\left.\mathbf{T}_{\widehat{\gamma}^{(k)}} \mathbf{d}_{\mathrm{exp}, n}\right)^{\mathrm{T}} \mathbf{r}_{M}}\right.
\end{array}\right]+\widehat{\mathbf{M}}^{(k)} \frac{\delta \mathbf{g}}{\delta \beta} \odot \mathbf{a}\left(\varphi_{\mathrm{rot}, n}^{(k)}, \theta_{\mathrm{rot}, n}^{(k)}\right)\right] \widehat{p}_{n}^{(k)},
\end{aligned}
$$

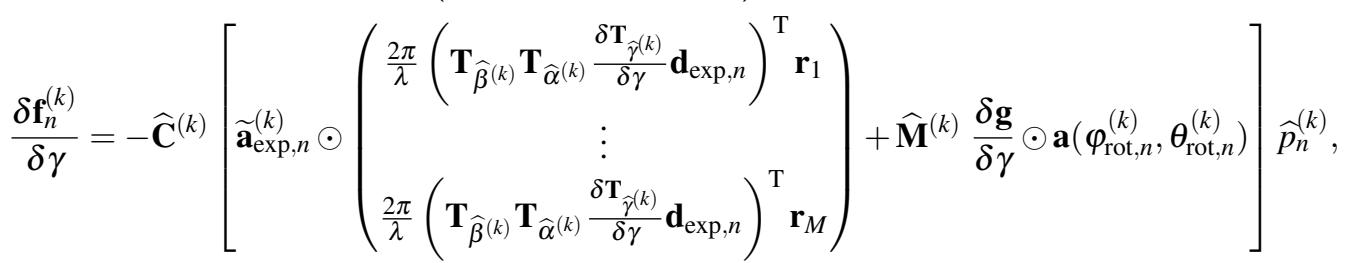

$$
\begin{aligned}
& \frac{\delta \mathbf{f}_{n}^{(k)}}{\delta c_{i}}=-\mathbf{J}^{(u, v)} \widetilde{\mathbf{a}}_{\mathrm{exp}, n}^{(k)} \widehat{p}_{n}^{(k)}, \\
& \frac{\delta \mathbf{f}_{n}^{(k)}}{\delta p_{i}}=-\widehat{\mathbf{C}}^{(k)} \widetilde{\mathbf{a}}_{\mathrm{exp}, n}^{(k)},
\end{aligned}
$$

where $\mathbf{J}^{(i, j)}$ is a single-entry Matrix with $\left[\mathbf{J}^{(i, j)}\right]_{i, j}=1$. The indices $u$ and $v$ are calculated as $u=\bmod (i-1, M)+1$ and $v=\left\lfloor\frac{i-1}{M}\right\rfloor+1$. Note that if the antenna characteristic $\mathbf{g}\left(\varphi_{n}, \theta_{n}\right)$ is similar for nearby DOAs, the derivatives $\frac{\delta \mathbf{g}}{\delta \alpha}, \frac{\delta \mathbf{g}}{\delta \beta}$ and $\frac{\delta \mathbf{g}}{\delta \gamma}$ can be neglected. In this case the actual values of the mutual coupling matrix $\widehat{\mathbf{M}}^{(k)}$ do not need to be known. The derivatives of the rotation matrices are derived as follows:

$$
\frac{\delta \mathbf{T}_{\widehat{\gamma}^{(k)}}}{\delta \gamma}=\left(\begin{array}{ccc}
-\sin \widehat{\gamma}^{(k)} & -\cos \widehat{\gamma}^{(k)} & 0 \\
\cos \widehat{\gamma}^{(k)} & -\sin \widehat{\gamma}^{(k)} & 0 \\
0 & 0 & 1
\end{array}\right), \frac{\delta \mathbf{T}_{\widehat{\beta}^{(k)}}}{\delta \beta}=\left(\begin{array}{ccc}
-\sin \widehat{\beta}^{(k)} & 0 & \cos \widehat{\beta}^{(k)} \\
0 & 1 & 0 \\
-\cos \widehat{\beta}^{(k)} & 0 & -\sin \widehat{\beta}^{(k)}
\end{array}\right), \frac{\delta \mathbf{T}_{\widehat{\alpha}^{(k)}}}{\delta \alpha}=\left(\begin{array}{ccc}
1 & 0 & 0 \\
0 & -\sin \widehat{\alpha}^{(k)} & -\cos \widehat{\alpha}^{(k)} \\
0 & \cos \widehat{\alpha}^{(k)} & -\sin \widehat{\alpha}^{(k)}
\end{array}\right)
$$

\section{REFERENCES}

[1] M. Sgammini, F. Antreich, L. Kurz, M. Meurer, and T. G. Noll, "Blind adaptive beamformer based on orthogonal projections for GNSS," in Proceedings of the 25th International Technical Meeting of the Satellite Division of the Institute of Navigation, Manassas, Va., September 2012.

[2] J. Arribas, C. Fernández-Prades, and P. Closas, "Multi-antenna techniques for interference mitigation in GNSS signal acquisition," EURASIP Journal on Advances in Signal Processing, vol. 2013, no. 1, p. 143, 2013. [Online]. Available: http://dx.doi.org/10.1186/1687-6180-2013-143

[3] L. Kurz and T. G. Zorn, Soeren Marcus und Noll, "Spatial spoofing signal suppression using the constellation covariance matrix," in Proceedings of the 29th International Technical Meeting of The Satellite Division of the Institute of Navigation, Portland, Oregon, USA, Sep. 2016, p. 30443052.

[4] M. Meurer, A. Konovaltsev, M. Appel, and M. Cuntz, "Direction-of-Arrival assisted sequential spoofing detection and mitigation," in Proceedings of the 2016 International Technical Meeting of The Institute of Navigation, Monterey, California, Jan. 2016. 
[5] S. Irteza, N. Murtaza, S. Caizzone, R. Stephan, and M. A. Hein, "Compact planar l-band antenna arrays with optimal diversity performance," in 2011 IEEE-APS Topical Conference on Antennas and Propagation in Wireless Communications, Sept 2011, pp. 512-515.

[6] A. A. Gheethan, P. A. Herzig, and G. Mumcu, "Compact 2 x 2 coupled double loop GPS antenna array loaded with broadside coupled split ring resonators," IEEE Transactions on Antennas and Propagation, vol. 61, no. 6, pp. 3000-3008, June 2013.

[7] S. Caizzone, G. Buchner, and W. Elmarissi, "Miniaturized dielectric resonator antenna array for GNSS applications," International Journal of Antennas and Propagation, no. Volume 2016, 2016. [Online]. Available: http://dx.doi.org/10.1155/2016/2564087

[8] S. Caizzone, "Miniaturized E5a/E1 antenna array for robust gnss navigation," IEEE Antennas and Wireless Propagation Letters, vol. 16, pp. 485-488, 2017.

[9] P. Sakian, R. Mahmoudi, and A. van Roermund, RF-Frontend Design for Process-Variation-Tolerant Receivers. Springer US, 2012. [Online]. Available: https://doi.org/10.1007/978-1-4614-2122-1

[10] A. Konovaltsev, M. Cuntz, L. A. Greda, M. V. T. Heckler, and M. Meurer, "Antenna and RF front end calibration in a GNSS array receiver," in 2010 IEEE International Microwave Workshop Series on RF Front-ends for Software Defined and Cognitive Radio Solutions (IMWS), Feb 2010, pp. 1-4.

[11] B. Friedlander and A. J. Weiss, "Direction finding in the presence of mutual coupling," IEEE Transactions on Antennas and Propagation, vol. 39, no. 3, pp. 273-284, Mar 1991.

[12] B. C. Ng and C. M. S. See, "Sensor-array calibration using a maximum-likelihood approach," IEEE Transactions on Antennas and Propagation, vol. 44, no. 6, pp. 827-835, Jun 1996.

[13] F. Sellone and A. Serra, "A novel online mutual coupling compensation algorithm for uniform and linear arrays," IEEE Transactions on Signal Processing, vol. 55, no. 2, pp. 560-573, Feb 2007.

[14] M. Lin, Z. Gong, and L. Yang, "A method for doa estimation with mutual coupling present," in 2005 IEEE International Symposium on Microwave, Antenna, Propagation and EMC Technologies for Wireless Communications, vol. 2, Aug 2005, pp. $926-935$.

[15] M. Wang, X. Ma, S. Yan, and C. Hao, "An autocalibration algorithm for uniform circular array with unknown mutual coupling," IEEE Antennas and Wireless Propagation Letters, vol. 15, pp. 12-15, 2016.

[16] Y. Wang, M. Trinkle, and B. W.-H. Ng, "DOA estimation under unknown mutual coupling and multipath with improved effective array aperture," Sensors, vol. 15, no. 12, p. 3085630869, dec 2015. [Online]. Available: https://www.ncbi.nlm.nih.gov/pmc/articles/PMC4721752/

[17] Z. XU, M. Trinkle, and D. A. Gray, "A maximum-likelihood based mutual coupling calibration algorithm in the presence of multipath for GPS antenna array," in Proceedings of the 24th International Technical Meeting of The Satellite Division of the Institute of Navigation. ION, 2011, pp. $1111-1119$.

[18] S. Zorn, M. Niestroj, M. Meurer, F. Wendler, and M. Cuntz, "Self-contained calibration determination by jointly solving the attitude estimation and calibration problem in the steering vector domain," in 2016 8th ESA Workshop on Satellite Navigation Technologies and European Workshop on GNSS Signals and Signal Processing (NAVITEC), Dec 2016, pp. 1-9.

[19] Z. M. Liu and Y. Y. Zhou, "A unified framework and sparse bayesian perspective for direction-of-arrival estimation in the presence of array imperfections," IEEE Transactions on Signal Processing, vol. 61, no. 15, pp. 3786-3798, Aug 2013.

[20] J. Dai, N. Hu, W. Xu, and C. Chang, "Sparse bayesian learning for doa estimation with mutual coupling," Sensors, vol. 15, no. 10, pp. 26267-26280, 2015. [Online]. Available: http://www.mdpi.com/1424-8220/15/10/26267

[21] P. B. Anantharamu, D. Borio, and G. Lachapelle, "Self-contained antenna array calibration using gnss signals," Journal of the Institute of Navigation, vol. 59, no. 3, pp. 209 - 220, 2012.

[22] W. Dahmen and A. Reusken, Numerik für Ingenieure und Naturwissenschaftler. Springer Berlin Heidelberg, 2008. [Online]. Available: https://doi.org/10.1007/978-3-540-76493-9 